\title{
Impacto de un programa de rehabilitación cardiovascular fase II sobre la calidad de vida de los pacientes
}

\author{
María José Bustamante ${ }^{1}$, Mónica Acevedo ${ }^{1}$, Giovanna Valentino ${ }^{1,2}$, \\ Cinthia Casasbellas ${ }^{1}$, María José Saavedra ${ }^{1}$, Marcela Adasme $^{1}$, Lorena Orellana ${ }^{1}$, Ismael Vergara ${ }^{1}$, \\ Ramón Corbalán ${ }^{1}$, Carlos Navarrete ${ }^{3}$.
}

\author{
1 Escuela de Medicina, Facultad de Medicina, División de Enfermedades Cardiovasculares. \\ 2 Unidad Docente Asociada de Ciencias de la Salud, Carrera de Nutrición. Facultad de Medicina, Pontificia \\ Universidad Católica de Chile. \\ 3 Departamento de Matemáticas, Universidad de La Serena.
}

Antecedentes: La rehabilitación cardiovascular (RCV) ha demostrado mejorar la capacidad física (CF) y la calidad de vida. La relación de estos aspectos ha sido escasamente reportada en Chile.

Objetivos: 1) Determinar el impacto de la RCV fase II sobre la calidad de vida, medida por la encuesta SF36 $y, 2$ ) determinar qué factores, relacionados con el paciente, pueden incidir en esta relación.

Método: Estudio prospectivo en sujetos que completaron 36 sesiones de un programa de RCV y Prevención Secundaria (PREV2). Se registraron al ingreso y final de la RCV: antecedentes médicos, factores de riesgo cardiovascular (CV), parámetros antropométricos, previsión de salud, CF (determinada por los metros caminados en test de marcha de 6 minutos-TM6) y puntaje en la encuesta SF36.

Resultados: 277 sujetos (78\% hombres, edad
59 años). Hubo una mejoría significativa en: a) los metros caminados (diferencia final/inicial= 56 metros, $\mathrm{p}<0.0001$ ), b) puntaje de salud física (68 vs 79; $\mathrm{p}<0.0001$ ), c) salud emocional (68 vs $78, \mathrm{p}<0.0001$ ) del SF36. Los hombres caminaron más metros promedio $(\mathrm{p}<0.0001)$. Hubo una asociación significativa entre los deltas del TM6 final-inicial y SF36 final-inicial, sólo para salud física $(\mathrm{p}<0.01)$. Por un aumento de 10 metros caminados en el TM6 final se incrementa el puntaje de la SF36 para salud física (Hombres: 0.38/ Mujeres: 1.52). La mejoría en la salud emocional sólo se asoció significativamente con los cambios en la salud física $(\mathrm{p}<0.0001)$.

Conclusión: Los pacientes que asisten a un programa de RCV mejoran significativamente su capacidad aeróbica, y su percepción de salud física y emocional.

\section{Correspondencia:}

Dra. Mónica Acevedo

División de Enfermedades Cardiovasculares

Pontificia Universidad Católica de Chile

Portugal 61, Santiago Centro, Santiago, Chile

Fono: 562-23543334

macevedo@med.puc.cl 


\section{Quality of life: impact of a phase II Cardiovascular Rehabilitation Program}

Background: Cardiovascular rehabilitation (CVR) has been shown to improve functional capacity (FC) and quality of life. The relation between these aspects has been scarcely reported in Chile.

Objectives: 1) To determine the impact of the CVR phase II program on quality of life as measured by the SF36 survey and 2) to determine which patient related factors can influence this relationship.

Method: Prospective study in subjects who completed 36 sessions of an RCV and Secondary Prevention program (PREV2). Medical records, cardiovascular risk factors (CV), anthropometric parameters, health forecast, functional capacity (FC) (determined by meters walked on 6-minute Walking test-TM6) and SF36 scores were recorded at the beginning and end of the CVR.

Results: Data on 277 subjects ( $78 \%$ men, age 59 years) was analyzed. There was a significant impro- vement in: a) walking meters (final / initial differen$\mathrm{ce}=56$ meters, $\mathrm{p}<0.0001)$, b) physical health score (68 vs 79, p <0.0001), c) emotional health (68 vs $78, \mathrm{p}<0.0001)$ of the SF36. The men walked more meters $(\mathrm{p}<0.0001)$. There was a significant association between delta TM6 final-initial and SF36 final-initial only for physical health $(\mathrm{p}<0.01)$. For an increase of 10 meters walked in the final TM6, SF36 score for physical health increased 0.38 points in men ad 1.52 in women. Improvement in emotional health was significantly associated only with changes in physical health $(\mathrm{p}<0.0001)$.

Conclusion: Patients attending a phase II cardiovascular rehabilitation program significantly improve their aerobic capacity, and their perception of physical and emotional health.

Key words: Cardiovascular rehabilitation; quality of life; secondary prevention. 


\section{Introducción:}

La RCV fase II es indicación clase IA en todas las guías internacionales de PREV2 ${ }^{1}$. Sus beneficios en la CF, como en calidad de vida, han sido ampliamente documentados $2,3,4$. El ejercicio físico aplicado en estos programas se relaciona con mejoría de la capacidad aeróbica y reducción de la mortalidad $\mathrm{CV}^{5,6,7}$.

Si bien la manera más objetiva de medir la capacidad aeróbica es a través del consumo de oxígeno máximo (VO2 max), existen pruebas submáximas validadas y muy utilizadas por su fácil aplicación y menor costo. El test de marcha de 6 minutos (TM6) es una de ellas. Estima la $\mathrm{CF}$, siendo un buen indicador de la tolerancia al ejercicio, reflejando actividades de la vida diaria y traduciendo la utilización de vías metabólicas aeróbicas ${ }^{8,9}$. Es una prueba válida y confiable en la población con patología cardíaca $^{10}$, y un factor pronóstico de morbi-mortalidad en esa población ${ }^{8,10}$. Por otro lado, la RCV genera importantes cambios en la calidad de vida de los pacientes. En trabajos randomizados, comparando instrumentos de intervención psicológica (psicoterapia) versus antidepresivos más ejercicio, se ha demostrado que esta última combinación es estadísticamente mejor que el apoyo psicoterápico por sí solo ${ }^{11}$. Por ello, medir el impacto de la RCV en la calidad de vida se ha transformado en un objetivo tan importante como la mejoría en la $\mathrm{CF}^{10,13,14}$. Se ha reportado que después de solo 6 semanas en RCV se mejora la calidad de vida, cambios que se mantienen hasta el año post $\mathrm{RCV}^{6}, 12$.

El Medical Outcomes Study Short Form 36 questionnaire (SF36) es uno de los instrumentos más utilizados para evaluar calidad de vida, y ha sido validado en dis-

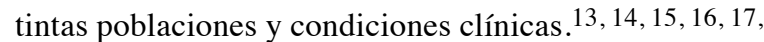
$18,19,20,22$.

Sin embargo, a pesar de la amplia evidencia respecto del impacto de la RCV sobre la calidad de vida, además de la mejoría en la $\mathrm{CF}$, no existen datos nacionales que den a conocer los resultados de este hecho. Probablemente, se debe a que la principal preocupación del grupo cardiológico es la cuantificación de la mejoría en la CF. Sin embargo, en la actualidad, la calidad de vida ha cobrado mayor importancia ya que la mejoría en el ámbito social y emocional mejora la adherencia de los pacientes a la terapia farmacológica, controles clínicos y a un estilo de vida saludable, que incluye el ejercicio mismo.

El objetivo del presente estudio es describir el impacto de un programa de RCV sobre la capacidad funcional y su impacto en la calidad de vida de sujetos en PREV2 que completaron las 36 sesiones del programa.

\section{Material y método:}

Se efectuó un estudio prospectivo en 277 pacientes que ingresaron al programa de RCV y PREV2 de un hospital universitario entre Octubre 2001 y Diciembre 2015.

\section{- Recolección de datos:}

Todos los sujetos fueron integrados a la Fase II de la RCV que dura 36 sesiones (aproximadamente 12 semanas). Se incluyeron pacientes en PREV2 y pacientes en prevención primaria, pero que clasificaran como equivalente coronario: diabéticos, insuficientes renales, pacientes con enfermedad aterosclerótica de territorio no coronario, y aquellos con puntaje de riesgo AHA $2013>7.5 \%$ (PREV1) o sea, pacientes de alto riesgo $\mathrm{CV}$. También se incluyeron pacientes valvulares operados, por cirugía tradicional. Se excluyeron todos aquellos pacientes que no completaron las 36 sesiones del programa. Esto, porque la evidencia es categórica en que los máximos beneficios de la RCV se consiguen después de las 24 semanas.

Se registraron en la ficha clínica los antecedentes mórbidos, factores de riesgo $\mathrm{CV}$, tipo de intervención $\mathrm{CV}$ (procedimiento percutáneo con stent, cirugía de revascularización coronaria y/o tratamiento médico óptimo), tipo de previsión de salud y años de estudio. Tanto al ingreso como a las 36 sesiones, se realizó un TM6 supervisado por kinesiólogo según protocolo $\mathrm{ATS}^{8}$, se aplicó la encuesta de calidad de vida SF36 y se midieron el índice de masa corporal (IMC), cintura, e índice de cintura-cadera. Todos los sujetos asistieron al programa 2 a 3 veces por semana.

La prescripción de ejercicio la realizó un cardiólogo basándose en los METs caminados y la frecuencia cardíaca alcanzada en una prueba de esfuerzo máxima graduada por síntomas, que se realiza a todos los pacientes que ingresan al programa. La rutina de ejercicio fue supervisada por un kinesiólogo y una enfermera, y consistió en: calentamiento previo, entrenamiento aeróbico en cicloergómetro, cinta ergométrica o elíptica, con una intensidad de 50 a $80 \%$ de la frecuencia cardíaca de reserva o el 60 a 85\% del VO2máx más ejercicios de sobrecarga al $70 \%$ de 1 repetición máxima, vuelta a la calma y flexibilidad. En cada sesión se monitorearon signos vitales en reposo y ejercicio y se evaluó la sensación subjetiva de fatiga con la Escala de Borg ${ }^{21}$ durante el ejercicio, para graduar su intensidad. Todos los sujetos recibieron educación programada acerca de sus FRCV, medicamentos, cambios de estilos de vida y planteamiento de sus objetivos en el programa.

- Definición de Factores de Riesgo Cardiovascular

Hipertensión: se consideró hipertensos a todos los suje- 
tos con diagnóstico médico de HTA, con o sin fármacos y a aquellos con más de 2 determinaciones de PA $\geq 140 / 90$ $\mathrm{mmHg}$.

Dislipidemia: sujetos con diagnóstico médico de hipercolesterolemia, con o sin tratamiento farmacológico, y aquellos con colesterol total $\geq 200 \mathrm{mg} / \mathrm{dL}$, y/o colesterol HDL $<40$ en hombres $\mathrm{y}<50 \mathrm{mg} / \mathrm{dL}$ en mujeres.

Obesidad: sujetos con IMC $\geq 30 \mathrm{y}$, obesos abdominales (cintura hombres $\geq 90$ y mujeres $\geq 80 \mathrm{~cm}$ ).

Diabetes: sujetos con diagnóstico médico de diabetes con o sin medicamentos, y aquellos con glicemia de ayuno $\geq 126$ $\mathrm{mg} / \mathrm{dL}$ en examen de ingreso; se consideró hiperglicemia si la glicemia de ayuno era $\geq 100 \mathrm{mg} / \mathrm{dL}$.

Historia familiar de cardiopatía: eventos coronarios precoces en uno o más familiares directos (hombre $<55$ y/o mujer $<65$ años).

\section{- Test de Marcha de 6 minutos:}

Se realizó según el protocolo de la American Thoracic Society $^{8}$. Los sujetos caminaron durante 6 minutos y se registraron los metros caminados, frecuencia cardíaca, presión arterial, saturación de oxígeno y frecuencia respiratoria basalmente y al término de la prueba.

\section{- Encuesta de Calidad de Vida}

Se aplicó de forma individual (auto aplicada) o, excepcionalmente, por la enfermera del programa en sujetos con discapacidad visual. La encuesta SF36 consta de 36 ítems que exploran 8 dimensiones del estado de salud: función física, función social, limitaciones del rol físico, rol emocional, salud mental, vitalidad, dolor y percepción de la salud general. El puntaje se obtiene codificando y promediando cada dimensión, obteniendo un valor entre 0 y 100, siendo esto directamente proporcional al estado de salud ${ }^{22}$. Para el análisis de los datos, las dimensiones se agruparon en dos áreas según las normas de SF36 de EEUU23.

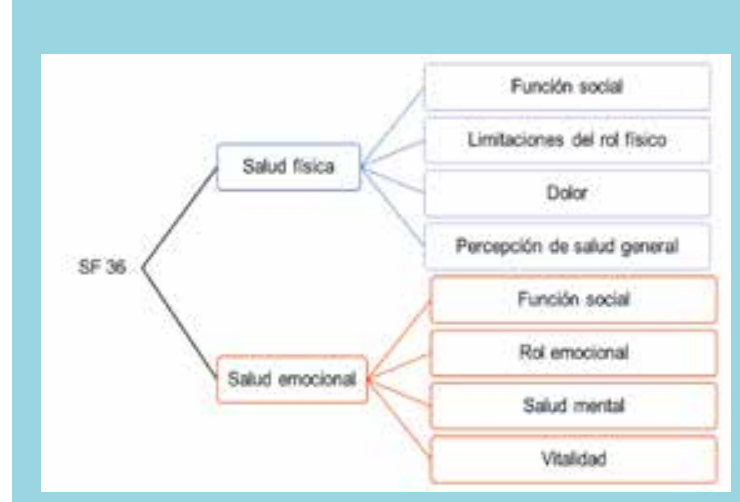

\section{Análisis estadístico:}

Se realizó estadística descriptiva para valores iniciales, finales y para la diferencia, tablas de contingencia con asociación analizada según test exacto de Fisher. Las hipótesis relacionadas con la mejoría después del programa de rehabilitación se probaron mediante test t pareados y modelos de regresión lineal.

\section{Resultados:}

De un universo de 1444 sujetos, 1167 no completaron las 36 sesiones del programa. En la Tabla 1, se puede ver que los pacientes que no terminaron las 36 sesiones, llamados no adherentes, eran más fumadores, menos dislipidémicos, más obesos, y tuvieron una menor prevalencia de cirugía cardíaca e intervenciones percutáneas comparado a los 277 que sí terminaron el programa (adherentes). Pero no hubo diferencias significativas entre los grupos en los promedios iniciales del TM6 y en la encuesta SF36.

De los 277 sujetos que completaron el programa, 70\% correspondía a sujetos de PREV2 $(\mathrm{N}=194)$ y el resto a PREV1. La prevalencia de factores de riesgo fue: Hipertensión arterial $60 \%$, Diabetes 14\%, tabaquismo 3\%, Dislipidemia $81 \%$, Obesidad 22\%, Sedentarismo $78 \%$ y antecedente familiar de cardiopatía coronaria $25 \%$. Las intervenciones post evento coronario fueron: angioplastía con stents $47 \%$, revascularización quirúrgica 37\% y tratamiento médico $2 \%$ (Tabla 1 ).

\begin{tabular}{|l|c|c|c|}
\hline $\begin{array}{l}\text { Tabla 1: Características demográficas, prevalencia de factores de riesgo } \\
\text { cardiovascular y promedios de puntaje de Encuesta SF } 36 \text { entre sujetos } \\
\text { adherentes y no adherentes al programa (2001 - 2015) }\end{array}$ \\
\hline & $\begin{array}{c}\text { Grupo no adherente } \\
(\mathrm{n}=1166)\end{array}$ & $\begin{array}{c}\text { Grupo adherente } \\
(\mathrm{n}=277)\end{array}$ & $\mathrm{P}$ \\
\hline Edad (años) & 57 & 58.5 & 0.05 \\
\hline Mujeres (\%) & $30 \%$ & $22 \%$ & $<0.01$ \\
\hline Hipertensión arterial (\%) & $55 \%$ & $60 \%$ & $\mathrm{NS}$ \\
\hline Diabetes (\%) & $13 \%$ & $14 \%$ & $\mathrm{NS}$ \\
\hline Tabaquismo (\%) & $13 \%$ & $3 \%$ & $<0.0001$ \\
\hline Dislipidemia (\%) & $66 \%$ & $81 \%$ & $<0.0001$ \\
\hline Obesidad (\%) & $30 \%$ & $22 \%$ & $<0.01$ \\
\hline Sedentarismo (\%) & $82 \%$ & $78 \%$ & $\mathrm{NS}$ \\
\hline Cirugía (\%) & $19 \%$ & $26 \%$ & 0.02 \\
\hline Angioplastía + Stent (\%) & $24 \%$ & $34 \%$ & $<0.01$ \\
\hline Tratamiento médico (\%) & $2 \%$ & $2 \%$ & $\mathrm{NS}$ \\
\hline SF36 física inicial & $20 \%$ & $18 \%$ & $\mathrm{NS}$ \\
\hline SF36 emocional inicial & $21 \%$ & $20 \%$ & $\mathrm{NS}$ \\
\hline Test de Marcha 6' inicial & $98 \%$ & $90 \%$ & $\mathrm{NS}$ \\
\hline
\end{tabular}




\begin{tabular}{|c|c|c|c|c|c|c|c|}
\hline & \multicolumn{2}{|c|}{ Mujeres } & \multicolumn{2}{|c|}{ Hombres } & \multicolumn{2}{|c|}{ Grupo Total } & \multirow{2}{*}{$\begin{array}{c}p \\
\text { Grupo total } \\
\text { (final v/s inicial }\end{array}$} \\
\hline & Inicial & Final & Inicial & Final & Inicia & Final & \\
\hline TM6 & $482 \pm 65$ & $530 \pm 66$ & $553 \pm 91$ & $609 \pm 96$ & $537 \pm 90$ & $591 \pm 96$ & $<0.0001$ \\
\hline Salud emocional & $62 \pm 22$ & $75 \pm 15$ & $69 \pm 19$ & $79 \pm 19$ & $68 \pm 20$ & $78 \pm 18$ & $<0.0001$ \\
\hline Salud física & $61 \pm 19$ & $75 \pm 16$ & $68 \pm 18$ & $81 \pm 13$ & $68 \pm 18$ & $80 \pm 14$ & $<0.0001$ \\
\hline
\end{tabular}

Hubo una alta prevalencia de factores de riesgo en los 277 sujetos que completaron el programa. Más del $50 \%$ tenían dislipidemia, sedentarismo o hipertensión. Una prevalencia menor se observó para diabetes, obesidad y tabaquismo. Las principales intervenciones fueron la angioplastía con stents y la cirugía de revascularización. Un mínimo de pacientes recibía solo tratamiento médico (Tabla 1).

$\mathrm{Al}$ analizar el grupo total de pacientes hubo una mejoría de $10 \%$ (promedio de 56.4 metros) en la distancia caminada del TM6, lo que fue estadísticamente significativo $(\mathrm{p}<0.0001)$. Tanto hombres como mujeres mejoraron la distancia, pero los hombres caminaron más metros que las mujeres, tanto al inicio como al final del programa, siendo estas diferencias significativas (Tabla 2).

El puntaje de la SF36 para salud emocional y salud física mejoró tanto en hombres como en mujeres en forma significativa. Sin embargo, el puntaje fue mayor para los hombres, tanto en la evaluación inicial, como en la evaluación final (Tabla 2). Al evaluar la SF36 para el grupo total de pacientes, se observó una diferencia de 10.5 puntos en salud emocional y de 11.6 puntos en salud física ( $\mathrm{p}<0.0001$, respectivamente).

$\mathrm{Al}$ asociar los resultados de la mejoría en la distancia caminada con los resultados de la SF36 (final-inicial), se observó que, por cada 10 metros de aumento en la distancia recorrida, se obtuvo una mejoría promedio de 0.38 puntos para los hombres y 1.52 puntos en las mujeres en el ámbito de la salud física $(p=0.01)$ (Figura 1). Al determinar los factores involucrados en el incremento de la capacidad aeróbica, la presencia de Hipertensión arterial determinó una menor distancia caminada al término de la RCV ( $\mathrm{p}=0.038)$.

Por otra parte, al determinar los factores involucrados en el incremento de la percepción de la salud física en el SF36, se encontró que sólo la mejoría en los metros caminados determinó una mejor salud física. Otros factores, como hipertensión, diabetes, obesidad o sedentarismo previo, no se

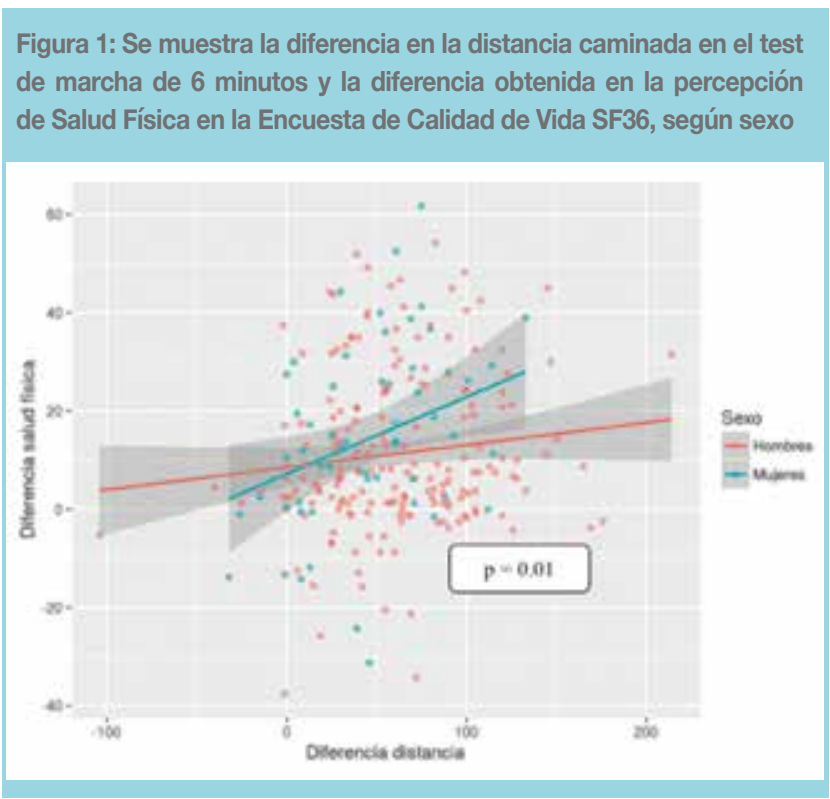

Figura 2: Se muestra la diferencia en la distancia caminada en el test de marcha de 6 minutos y la diferencia obtenida en la percepción de Salud Emocional en la Encuesta de Calidad de Vida SF36, según sexo

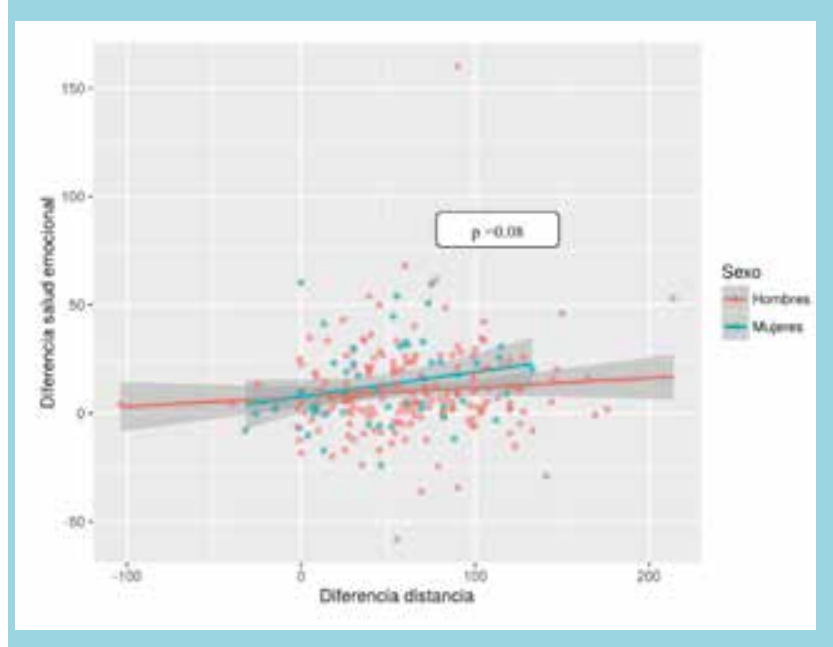


relacionaron con esta mejoría.

Por último, al determinar los factores involucrados en el incremento de la salud emocional, sólo se encontró que la mejoría de la percepción en salud física determinó una mejor salud emocional ( $\mathrm{p}=0.0001)$ (Figura 3). El aumento en la distancia caminada mostró solo una tendencia a correlacionarse con mejor salud emocional $(\mathrm{p}=0.08)$ (Figura 2).

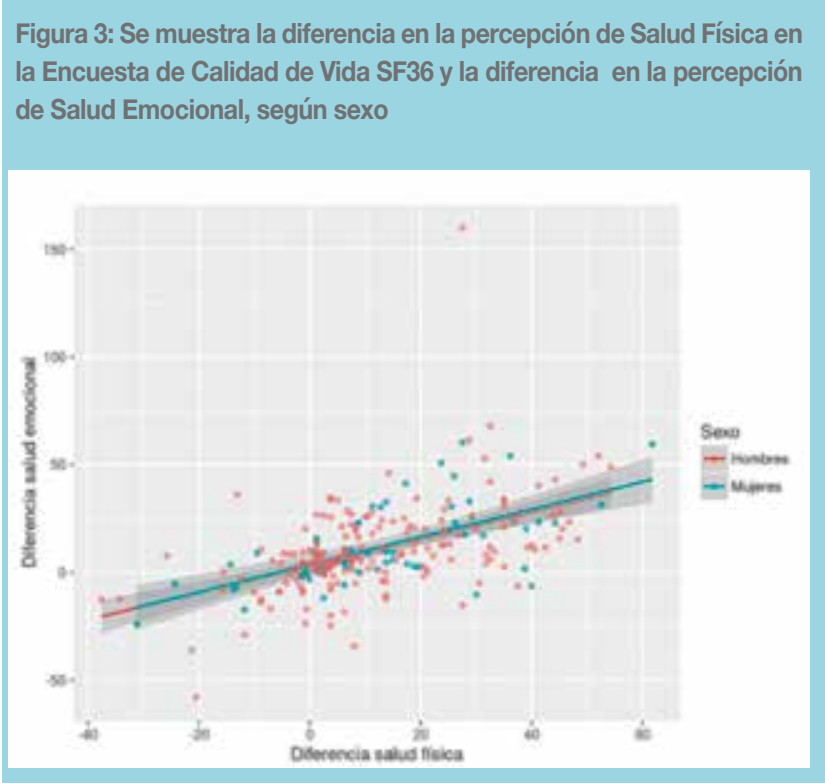

\section{Discusión}

Los resultados de este estudio demuestran que, tanto en PREV1 como en PREV2 los sujetos que han completado el programa de RCV, es decir 36 sesiones, mejoran su capacidad aeróbica y calidad de vida. Estos resultados son los primeros a nivel nacional que reportan los beneficios de la RCV más allá de la capacidad física. Se encontró una mejoría significativa, de un $10 \%$, en el rendimiento de la distancia caminada en el TM6 en el grupo total. Si bien, el mejor método para valorar la mejoría de la capacidad aeróbica es con la medición del VO2max en un test cardiopulmonar, o al menos, con una prueba de esfuerzo máxima, en nuestro país esto no se hace aún de rutina, pues la RCV no es cubierta como tal en los planes de salud privados ni públicos. Por ello, la mayoría de los centros nacionales evalúan el progreso en la capacidad aeróbica con el TM6. El incremento obtenido en nuestro estudio en la distancia caminada fue menor que el reportado en la literatura. Como ejemplo, Verril y cols., demostraron un aumento de $15 \%^{2}$. Esto no es una sorpresa. La mejoría en la capacidad aeróbica está di- rectamente relacionada a la capacidad funcional del paciente al entrar al programa. Está demostrado que los pacientes con peor capacidad funcional son los que más mejoran con la RCV, mejoría que puede llegar a 30\%. La menor mejoría demostrada en nuestros pacientes se puede explicar porque, en general, aún existe reticencia de parte del profesional médico en derivar a los sujetos que tienen una condición física deteriorada. Por ello, la mayoría de los pacientes derivados son aquellos que insisten en querer reanudar la actividad física. Esta situación debiera mejorar en los próximos años ya que, como objetivo estratégico 2011-2020 el Ministerio de Salud de Chile propuso la incorporación de la RCV después de un infarto.

El aporte de este trabajo, sin embargo, no está en la mejoría de la capacidad aeróbica, que era esperable, sino en la mejoría en la calidad de vida, tanto en su esfera de percepción de salud física como emocional. Desde el inicio de nuestro programa de RCV se aplica la encuesta SF36. Esta nos ha permitido derivar pacientes con trastornos depresivos al especialista. Esta encuesta es analizada por la enfermera del programa, quién evalúa la necesidad de derivación a través del análisis objetivo de los puntajes, y previo aviso al médico tratante. Nuestros resultados demostraron una clara mejoría de ambos aspectos en la calidad de vida del grupo total: dimensión de salud física como emocional, lo que concuerda con datos documentados en la literatura ${ }^{25}$. El principal factor determinante de la mejoría en la salud física fue la mejoría en la capacidad aeróbica: todos los pacientes al ejercitarse "perciben" que están mejor en lo físico. Sin embargo, también observamos una tendencia (pero no significativa, $\mathrm{p}=0.08$ ) a que la mejoría en la distancia caminada se asociara a una mejoría en la salud emocional. Probablemente, la no significancia estuvo dada porque la mejoría en la salud física fue la que tuvo más impacto en la mejoría de la salud emocional, y no el incremento en la distancia caminada. Pero demostrar esto es muy difícil, ya que la mejoría de la salud física y el incremento en la distancia objetiva caminada ocupan las mismas vías fisiológicas y una enmascara a la otra en modelos multivariados.

Finalmente, es importante destacar que, a pesar de que el grupo de mujeres fue pequeño, también presentaron buenos resultados (ver Figura 1, 2 y 3 ). Estas, en general, son menos derivadas a los programas de RCV. Esto ha sido demostrado, no sólo por nuestro grupo ${ }^{20}$, sino destacado en las guías de RCV de la Sociedad Europea de Cardiología 24 . Los factores que influyen en esto incluyen a profesionales médicos que no derivan, reticencia de las mismas pacientes a acudir al programa, percepción de los profesionales de la salud de que las mujeres no tienen enfermedad coronaria severa. 
Nuestros resultados tienen importancia porque se ha demostrado que uno de los principales factores de fracaso en la PREV2 es la falta de adherencia.

La mejoría de la calidad de vida podría mejorar la adherencia de los pacientes no sólo a medicamentos, sino también a adherir a conductas más saludables. Esto, no ha sido reportado en la literatura. En un estudio nuestro reciente, demostramos que la adherencia al programa era muy baja $(30 \%)^{20}$, y que el puntaje SF36 condicionó la baja adherencia a la RCV. De allí lo valioso de los resultados del presente estudio: una mejor calidad de vida posterior a RCV podría traducirse en mejorar todos los factores involucrados en la PREV2, lo que deberá demostrarse en futuros estudios a nivel nacional. Así, el presente reporte resulta generador de una hipótesis no respondida: "La mejoría en la capacidad aeróbica y calidad de vida de los pacientes en PREV2 después de un programa de
$\mathrm{RCV}$, ¿podría mejorar la adherencia a medicamentos y/o la adherencia a conductas más saludables?

Nuestro estudio tiene limitaciones: alto porcentaje de abandono del programa, lo que nos expone a un número pequeño de sujetos incluidos en esta investigación. Un $79 \%$ de los sujetos de RCV no completaron el programa, lo que lo que supera lo reportado en otros estudios $(67 \%, 42 \%$ y $11 \% 26$, 27,28 ) y este grupo de pacientes obtuvo puntajes de SF36 basales menores que el grupo adherente, lo que podría reflejar una menor motivación para el cambio de hábitos.

Si bien todos los sujetos ingresados al programa son sometidos a una prueba de esfuerzo inicial, no contamos con una nueva prueba, lo que habría permitido una mejor evaluación de la capacidad física. Finalmente, se trata de una población seleccionada, relativamente joven y la mayoría en capacidad funcional II.

\section{Referencias}

1. VAN DE WERF F, BAX J, BETRIU A, BLOMSTROM-LUNDQVIST C, CREA F, FALK V, et al. Management of acute myocardial infarction in patients presenting with persistent st-segment elevation: The task force on the management of st-segment elevation acute myocardial infarction of the european society of cardiology. Eur Heart J. 2008; 29: 2909-2945.

2. VERRILL D, BARTON C, BEASLEY W, LIPPARD M, KING CN, DAVISON C. Six-minute walk performance and quality of life comparisons in North Carolina cardiac rehabilitation programs. Heart \& lung. 2003; 32: 41-51.

3. American Association of Cardiovascular and Pulmonary Rehabilitation. Guidelines for cardiac rehabilitation and secondary prevention programs. 3rd ed. Champaign (IL): Human Kinetics. 1999; 185-7.

4. VERRILL D, BARTON C, BEASLEY W, BRENNAN M, LIPPARD M, KING C. Quality of life measures and gender comparisons in North Carolina cardiac rehabilitation programs. J Cardiopulm Rehabil. 2001; 21:37-46.
5. WENGER NK. Current status of cardiac rehabilitation. J Am Coll Cardiol. 2008; 51;1619-1631.

6. YOHANNES A, DOHERTY P, BUNDY C, YALFANI A. the long-term benefits of cardiac rehabilitation on depression, anxiety, physical activity and quality of life. Journal of Clinical Nursing. 2010; 19:2806-2813.

7. THOMPSON PD, BUCHNER D, PINA IL, BALADY GJ, WILLIAMS MA, MARCUS BH, et al. Exercise and physical activity in the prevention and treatment of athero- sclerotic cardiovascular disease: a statement from the Council on Clinical Cardiology (Subcommittotal energy expenditure on Exercise, Rehabilitation and Prevention) and the Council on Nutrition, Physical Activity and Metabolism (Subcommittotal energy expenditure on Physical Activity). Circulation. 2003; 107; 3109-3116.

8. ATS Committee on Proficiency Standards for Clinical Pulmonary Function Laboratories. ATS statement: guidelines for the six-minute walk test. Am J Respir Crit Care Med 2002;166:111-117 
9. SOLWAY S, BROOKS D, LACASSE Y, THOMAS S. A qualitative systematic overview of the measurement properties of functional walk tests used in the cardiorespiratory domain. Chest. 2001;119:256-270

10. HAMILTON DM, HAENNEL RG. Validity and reliability of the 6-minute walk test in a cardiac rehabilitation population. $\mathrm{J}$ Cardiopulmonary Rehabilitation 2000; 20: 156-64.

11. BLUMENTHAL JA, BABYAK M, CARNEY R, HUBER M, SAAB P, BURG M, et al. Exercise, depression, and mortality after myocardial infarction in the ENRICHD trial. Med Sci Sports Exerc. 2004; 36: 746-55.

12. OLDRIDGE N, PERKINS A, MARCHIONNI N, FUMAGALLI S, FATTRIOLLI F, GUYATT G. Number needed to treat in cardiac rehabilitation. Journal of Cardiopulmonary Rehabilitation. 2002; 22: 22-30.

13. JOLLIFFE JA, REES K, TAYLOR R, THOMPSON D, OLDRIDGE N, EBRAHIM S. Exercise-based rehabilitation for coronary heart disease. The Cochrane Library. 2001.

14. MÜLLER-NORDHORN J, KULIG M, BINTING S, VOLLER H, GOHLKE H, LINDE K, et al. Change in quality of life in the year following cardiac rehabilitation. Qual Life Res. 2004; 13: 399-410.

15. DEMPSTER M, DONNELLY M. Measuring the health related quality of life of people with ischaemic heart disease. Heart. 2000; 83:641-644.

16. IZAWA K, HIRANO Y, YAMADA S, OKA K, OMIYA K, IIJIMA S. Improvement in physiological outcomes and health-related quality of life following cardiac rehabilitation in patients with acute myocardial infarction. Circulation Journal. 2004; 68:315-320.

17. HEVEY D, MCGEE HM, HORGAN J. Responsiveness of health-related quality of life outcome measures in cardiac rehabilitation: comparison of cardiac rehabilitation outcome measures. J Consult Clin Psychol. 2004;72:1175.

18. WARE J, SNOW K, KOSINSKI M, GANDEK B. Short form 36 health survey manual and interpretation guide. Boston: New England Medical Health Centre, The Health Institute; 1993.

19. DEVON HA, FERRANS CE. The psychometric properties of four quality of life instruments used in cardiovascular populations. J Cardiopulmonar Rehabilitation 2003; 23:122-138.

20. BUSTAMANTE M, VALENTINO G, KRÄMER V, ADASME M, GUIDI D, IBARA C, et al. Patient Adherence to a Cardiovascular Rehabilitation Program: What Factors Are Involved? International Journal of Clinical Medicine. 2015; 6: 605-614.

21. Borg G. Borg`s perceived exertion and pain scales. Champaign, IL: Human Kinetics, 1998 (<a ref="http://www.thefreelibrary.com/Borg's+Perceived+Exertion+and+Pain+Scales.-a053509933">Borg's Perceived Exertion and Pain Scales.</a $>$ ).

22. WARE JR JOHN, KOSINSKI M, KELLER S. A 12-Item Short-Form Health Survey: construction of scales and preliminary tests of reliability and validity. Medical care.1996;34: 220-233.

23. U.S. Population Norms. United States of America. 1998. http://www.sf-36.org/research/sf98norms.pdf

24. BJARNASON-WEHRENS B, MCGEE H, ZWISLER AD, PIEPOLI MF, BENZER W, SCHMID JP, et al. Cardiac Rehabilitation Section European Association of Cardiovascular Prevention and Rehabilitation. Cardiac rehabilitation in Europe: results from the European Cardiac Rehabilitation Inventory Survey. Eur J Cardiovasc Prev Rehabilitation. 2010; 17:410-418.

25. MCKEE G. Are there meaningful longitudinal changes in health related quality of life - SF 36, in cardiac rehabilitation patients? Eur J of Cardiovascular Nursing. 2009; 8:40-47.

26. SIN M-K, SANDERSON B, WEAVER M, GIGER J, PEMBERTON J, KLAPOW J. Personal characteristics, health status, physical activity, and quality of life in cardiac rehabilitation participants. International Journal of Nursing Studies. 2004;41:173-181.

27. SANDERSON BK, PHILLIPS MM, GERALD L, DILILLO V, BITTNER V. Factors associated with the failure of patients to complete cardiac rehabilitation for medical and nonmedical reasons. J Cardiopulm Rehabil. 2003; 23: 281-89.

28. JENNINGS S, CAREY D. Capacity and equity in cardiac rehabilitation in the eastern region: good and bad news. Ir J Med Sci. 2004; 173: 151-154. 